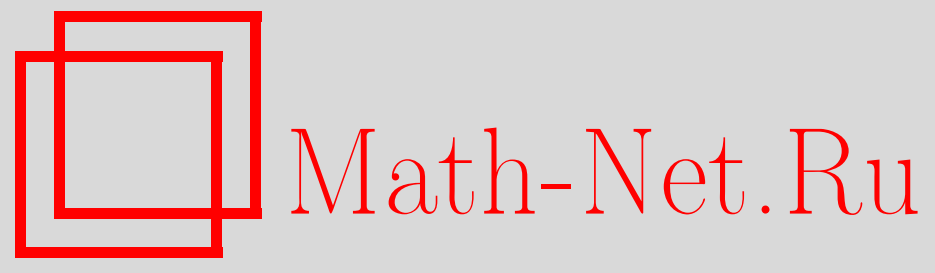

М. А. Сагадеева, А. Н. Шулепов, Задачи оптимального и жесткого управления решениями специального вида нестационарных уравнений соболевского типа, Вестн. Сам. гос. техн. ун-та. Сер. Физ.мат. науки, 2014, выпуск 2(), 33-38

DOI: https://doi.org/10.14498/vsgtu1286

Использование Общероссийского математического портала MathNet.Ru подразумевает, что вы прочитали и согласны с пользовательским соглашением

http://www.mathnet.ru/rus/agreement

Параметры загрузки:

IP : 3.81 .55 .215

26 апреля 2023 г., 14:26:00

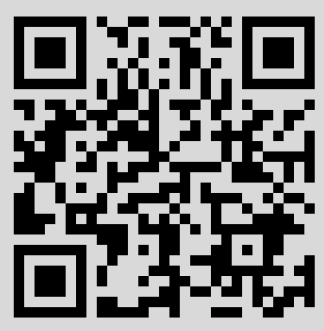


Вестн. Сам. гос. техн. ун-та. Сер. Физ.-мат. науки. 2014. № 2 (35). С. $33-38$

УДК 517.977.5

\title{
ЗАДАЧИ ОПТИМАЛЬНОГО И ЖЁСТКОГО УПРАВЛЕНИЯ РЕШЕНИЯМИ СПЕЦИАЛЬНОГО ВИДА НЕСТАЦИОНАРНЫХ УРАВНЕНИЙ СОБОЛЕВСКОГО ТИПА
}

\author{
М. А. Сагадеева, А. Н. Шулепов
}

Южно-Уральский государственный университет (национальный исследовательский университет), Россия, 454080, Челябинск, пр. Ленина, 76.

\begin{abstract}
Уравнения соболевского типа в настоящее время составляют обширную область среди неклассических уравнений математической физики. Неклассическими называют те уравнения математической физики, чви представления в виде уравнений или систем уравнений в частных производных не укладываются в рамки одного из классических типов - эллиптического, параболического или гиперболического. В данной работе показано существование единственного оптимального и жёсткого управлений решениями задачи Шоуолтера-Сидорова для нестационарного операторно-дифференциального уравнения, неразрешенного относительно производной по времени. Нестационарность уравнения рассмотрена в виде произведения одного из операторов уравнения и скалярной функиии, зависящей от времени, а свойства операторов таковы, что стационарное уравнение обладает разрешающей сильно непрерывной вырожденной полугруппой. Статъя, кроме введения и списка литературы, содержит две части. В первой части приводятся необходимые сведения теории относительно р-радиальных операторов, во второй части содержится основной результат статьи.
\end{abstract}

Ключевые слова: оптимальное управление, жёсткое управление, нестационарные уравнения соболевского типа, относительно радиальный случай.

Введение. Пусть $\mathfrak{X}, \mathfrak{Y}$ и $\mathfrak{U}$ - гильбертовы пространства. Пусть операторы $L \in \mathcal{L}(\mathfrak{X} ; \mathfrak{Y}), M \in \mathcal{C} l(\mathfrak{X} ; \mathfrak{Y})$ и $B \in \mathcal{L}(\mathfrak{U} ; \mathfrak{Y})$. Рассмотрим задачу ШоуолтераСидорова [1]

$$
P\left(x(0)-x_{0}\right)=0
$$

для уравнения соболевского типа [2-4]

$$
L \dot{x}(t)=a(t) M x(t)+f(t)+B u(t) \quad(\operatorname{ker} L \neq\{0\}), \quad t \in[0, \tau],
$$

где вектор-функции $u:[0, \tau] \rightarrow \mathfrak{U}, f:[0, \tau] \rightarrow \mathfrak{Y}$ и скалярная функция $a:[0, \tau] \rightarrow \mathbb{R}_{+}$будут определены в дальнейшем.

ISSN: 2310-7081 (online), 1991-8615 (print); doi: http://dx.doi.org/10.14498/vsgtu1286 (C) 2014 Самарский государственный технический университет.

Образец цитирования: М. А. Сагадеева, А. Н. Шулепов, “Задачи оптимального и жёсткого управления решениями специального вида нестационарных уравнений соболевского типа" // Вестн. Сам. гос. техн. ун-та. Сер. Физ.-мат. науки, 2014. № 2 (35). C. 33-38. doi: 10.14498/vsgtu1286.

Сведения об авторах: Минзиля Алмасовна Сагадеева (к.ф.-м.н., доцент), доцент, каф. информационно-измерительной техники. Андрей Николаевич Шулепов, магистрант, каф. уравнений математической физики.

E-mail addresses: sagadeeva_ma@mail.ru (M.A. Sagadeeva, Corresponding author), andrewn92@mail.ru (A.N. Shulepov) 
Нашей задачей является нахождение минимума функционала вида

$$
\begin{aligned}
J(u)=\alpha \sum_{q=0}^{1} \int_{0}^{\tau} \| z^{(q)}(t)-z_{d}^{(q)}(t) & \|_{\mathfrak{Z}}^{2} d t+ \\
& +(1-\alpha) \sum_{q=0}^{k} \int_{0}^{\tau}\left\langle N_{q} u^{(q)}(t), u^{(q)}(t)\right\rangle_{\mathfrak{U}} d t,
\end{aligned}
$$

где $\alpha \in(0,1], k=0,1, \ldots, p+1, N_{q} \in \mathcal{L}(\mathfrak{U})$ - самосопряжённые и положительно определённые операторы, $z=C x$, оператор $C \in \mathcal{L}(\mathfrak{X} ; \mathfrak{Z})$, а $z_{d}=z_{d}(t, s)$ плановое наблюдение из некоторого гильбертова пространства наблюдений $\mathfrak{Z}$. Отметим, что $\alpha \in(0,1]$ и $(1-\alpha)$ - весовые коэффициенты целей оптимального управления, заключающиеся в достижении плановых показателей наблюдаемой величины без скачкообразных изменений (первое слагаемое в (3)) и минимизации расходуемых для этого ресурсов управления (второе слагаемое в (3)). При $\alpha=1$ в функционале (3) исчезает второе слагаемое и мы получаем задачу жёсткого управления, т.е. когда при оптимизации затраты на достижение цели не интересны.

1. Относительно $\boldsymbol{p}$-радиальные операторы. Доказательства утверждений этой части можно найти в работе [3].

Обозначим

$$
\begin{gathered}
\rho^{L}(M)=\left\{\mu \in \mathbb{C}:(\mu L-M)^{-1} \in \mathcal{L}(\mathfrak{F} ; \mathfrak{U})\right\}, \quad \sigma^{L}(M)=\mathbb{C} \backslash \rho^{L}(M), \\
R_{\mu}^{L}(M)=(\mu L-M)^{-1} L, \quad L_{\mu}^{L}(M)=L(\mu L-M)^{-1}, \quad \mu \in \rho^{L}(M), \\
R_{(\lambda, p)}^{L}(M)=\prod_{k=0}^{p} R_{\lambda_{k}}^{L}(M), L_{(\lambda, p)}^{L}(M)=\prod_{k=0}^{p} L_{\lambda_{k}}^{L}(M), \quad \lambda_{k} \in \rho^{L}(M)(k=\overline{0, p}) .
\end{gathered}
$$

Определение 1. Оператор $M$ называется $p$-радиалъным относительно оператора $L$ (или, коротко, $(L, p)$-радиалънъмм), если

(i) $\exists \omega \in \mathbb{R} \forall \mu>\omega \Rightarrow \mu \in \rho^{L}(M)$;

(ii) $\exists K>0 \forall \mu_{k}>\omega, k=\overline{0, p}, \forall n \in \mathbb{N}$

$$
\max \left\{\left\|\left(R_{(\mu, p)}^{L}(M)\right)^{n}\right\|_{\mathcal{L}(\mathfrak{X})},\left\|\left(L_{(\mu, p)}^{L}(M)\right)^{n}\right\|_{\mathcal{L}(\mathfrak{Y})}\right\} \leqslant \frac{K}{\prod_{k=0}^{p}\left(\mu_{k}-\omega\right)^{n}} .
$$

Также введём обозначения

$$
\mathfrak{X}^{0}=\operatorname{ker} R_{(\mu, p)}^{L}(M), \mathfrak{Y}^{0}=\operatorname{ker} L_{(\mu, p)}^{L}(M), L_{0}=\left.L\right|_{\mathfrak{X}^{0}}, M_{0}=\left.M\right|_{\operatorname{dom} M \cap \mathfrak{X}^{0}} .
$$

Через $\mathfrak{X}^{1}\left(\mathfrak{Y}^{1}\right)$ обозначим замыкание линеала $\operatorname{im} R_{(\mu, p)}^{L}(M)\left(\operatorname{im} L_{(\mu, p)}^{L}(M)\right)$, а через $\tilde{\mathfrak{X}}(\tilde{\mathfrak{Y}})$ - замыкание линеала $\mathfrak{X}^{0} \dot{+} \operatorname{im} R_{(\mu, p)}^{L}(M)\left(\mathfrak{Y}^{0} \dot{+} \operatorname{im} L_{(\mu, p)}^{L}(M)\right)$ в норме пространства $\mathfrak{X}(\mathfrak{Y})$.

ОпредЕЛЕНие 2 . Сильно непрерывное отображение $V^{\bullet}: \mathbb{R}_{+} \rightarrow \mathcal{L}(\mathcal{V})$ называется сильно непрерьвной полугруппой разрешающих операторов (или просто разрешающей $C_{0}$-nолугруппой), если 
(i) $V^{s} V^{t}=V^{s+t} \forall s, t>0$;

(ii) $v(t)=V^{t} v_{0}$ есть решение этого уравнения для любого $v_{0}$ из плотного в $\mathcal{V}$ линеала.

Полугруппу $\left\{V(t) \in \mathcal{L}(\mathcal{V}): t \in \overline{\mathbb{R}}_{+}\right\}$будем называть экспоненииально ограниченной с константами $C, \omega$, если

$$
\exists C>0 \quad \exists \omega \in \mathbb{R} \quad \forall t \in \overline{\mathbb{R}}_{+} \quad\|V(t)\|_{\mathcal{L}(\mathcal{V})} \leqslant C e^{\omega t} .
$$

Теорема 1. Пусть оператор $M(L, p)$-радиален. Тогда существует экспоненииально ограниченная с константами $K, \omega$ из определения 1 и сильно непрерывная разрешающая полугруппа для однородного уравнения (2) при $a \equiv 1$, рассматриваемого на подпространстве $\tilde{\mathfrak{X}}$.

ЗАмечАниЕ 1. Операторы разрешающей полугруппы для уравнения (2) при $a \equiv 1$ и $t>0$ можно представить в виде

$$
X^{t}=s-\lim _{k \rightarrow \infty}\left(\left(L-\frac{t}{k} M\right)^{-1} L\right)^{k}=s-\lim _{k \rightarrow \infty}\left(\frac{k}{t} R_{\frac{k}{t}}^{L}(M)\right)^{k},
$$

принимая во внимание поправки формулы, обсуждаемые в работе [5].

ЗАмЕЧАниЕ 2. Единицей полугруппы $\left\{X^{t} \in \mathcal{L}(\tilde{\mathfrak{X}}): t \in \overline{\mathbb{R}}_{+}\right\}$является проектор $P$ вдоль $\mathfrak{X}^{0}$ на $\mathfrak{X}^{1}$.

ОПреДЕЛЕниЕ 3. Оператор $M$ называется силъно $(L, p)$-радиалъным, если при любых $\lambda, \mu_{0}, \mu_{1}, \ldots, \mu_{p}>\omega$ выполняются следующие условия:

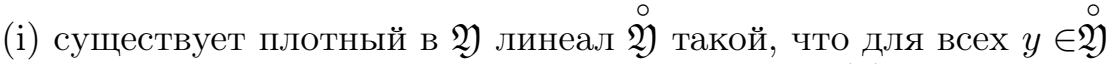

$$
\begin{gathered}
\left\|M(\lambda L-M)^{-1} L_{(\mu, p)}^{L}(M) y\right\|_{\mathfrak{Y}} \leqslant \frac{\operatorname{const}(y)}{(\lambda-\omega) \prod_{k=0}^{p}\left(\mu_{k}-\omega\right)} ; \\
\left\|R_{(\mu, p)}^{L}(M)(\lambda L-M)^{-1}\right\|_{\mathcal{L}(\mathfrak{Y} ; \mathfrak{X})} \leqslant \frac{K}{(\lambda-\omega) \prod_{k=0}^{p}\left(\mu_{k}-\omega\right)} .
\end{gathered}
$$

Теорема 2. Пусть оператор $M$ сильно $(L, p)$-радиален. Тогда

(i) $\mathfrak{X}=\mathfrak{X}^{0} \oplus \mathfrak{X}^{1}, \mathfrak{Y}=\mathfrak{Y}^{0} \oplus \mathfrak{Y}^{1}$;

(ii) $L_{k}=\left.L\right|_{\mathfrak{X}^{k}} \in \mathcal{L}\left(\mathfrak{X}^{k} ; \mathfrak{Y}^{k}\right), \quad M_{k}=\left.M\right|_{\text {dom } M_{k}} \in \mathcal{C l}\left(\mathfrak{X}^{k} ; \mathfrak{Y}^{k}\right)$, $\operatorname{dom} M_{k}=\operatorname{dom} M \cap \mathfrak{X}^{k}, k=0,1$;

(iii) существуют операторъ $M_{0}^{-1} \in \mathcal{L}\left(\mathfrak{Y}^{0} ; \mathfrak{X}^{0}\right)$ u $L_{1}^{-1} \in \mathcal{L}\left(\mathfrak{Y}^{1} ; \mathfrak{X}^{1}\right)$.

2. Оптимальное и жёсткое управления решениями задачи ШоуолтераСидорова для нестационарного уравнения

ОПРЕДЕЛЕНИЕ 4. Вектор-функция

$$
x \in H^{1}(\mathfrak{X})=\left\{x \in L_{2}(0, \tau ; \mathfrak{X}): \dot{x} \in L_{2}(0, \tau ; \mathfrak{X})\right\}
$$

называется сильным решением уравнения (2), если она почти всюду на $(0, \tau)$ обращает его в тождество. Сильное решение $x=x(t)$ уравнения (2) называется сильным решением задачи Шоуолтера-Сидорова (1), (2), если оно удовлетворяет (1). 
Обозначим $\mathbb{N}_{0} \equiv \mathbb{N} \cup\{0\}$. Построим гильбертово пространство

$$
H^{p+1}(\mathfrak{Y})=\left\{y \in L_{2}(0, \tau ; \mathfrak{Y}): y^{(p+1)} \in L_{2}(0, \tau ; \mathfrak{Y}), p \in \mathbb{N}_{0}\right\}
$$

со скалярным произведением

$$
[y, z]=\sum_{q=0}^{p+1} \int_{0}^{\tau}\left\langle y^{(q)}, z^{(q)}\right\rangle_{\mathfrak{Y}} d t .
$$

Теорема 3. Пусть оператор $M$ сильно $(L, p)$-радиален, $p \in \mathbb{N}_{0}$, а функиия $a \in C^{p+1}\left([0, \tau) ; \mathbb{R}_{+}\right)$. Тогда для любых $x_{0} \in \mathfrak{X} u f \in H^{p+1}(\mathfrak{Y})$ сушествует единственное сильное решение $x \in H^{1}(\mathfrak{X})$ задачи Шоуолтера-Сидорова (1) для уравнения (2), причём

$$
\begin{array}{r}
x(t)=X_{0}^{t} a(\zeta) d \zeta P x_{0}+\int_{0}^{t} X_{s}^{t} a(\zeta) d \zeta L_{1}^{-1} Q(f(s)+B u(s)) d s- \\
-\sum_{k=0}^{p}\left(M_{0}^{-1} L_{0}\right)^{k} M_{0}^{-1}(I-Q)(A D)^{k} A(f(t)+B u(t)),
\end{array}
$$

¿əe

$$
(A h)(t)=a^{-1}(t) h(t), \quad(D h)(t)=\frac{d h}{d t}(t) .
$$

Д о каз а m e ль с $m$ в о этой теоремы аналогично доказательству теоремы 4 из работы [6] и потому не приводится.

ОпредЕлЕниЕ 5. Вектор-функцию $v \in H_{\partial}^{1}(\mathfrak{U})$ назовем оптимальным управлением (жестким управлением) решениями задачи (1), (2), если

$$
J(v)=\min _{(x, u) \in \mathfrak{X} \times H_{\partial}^{1}(\mathfrak{U})} J(u), \quad \alpha \in(0,1) \quad(\alpha=1),
$$

где пары $(x, u) \in \mathfrak{X} \times H_{\partial}^{1}(\mathfrak{U})$ удовлетворяют $(1),(2)$, а $H_{\partial}^{1}(\mathfrak{U})-$ некоторое замкнутое и выпуклое подмножество в $H^{1}(\mathfrak{U})$.

Теорема 4. Пусть оператор $M$ сильно $(L, p)$-радиален, $p \in \mathbb{N}_{0}$, функиия $a \in C^{p+1}\left(\overline{\mathbb{R}}_{+} ; \mathbb{R}_{+}\right)$. Тогда при любъх $x_{0} \in \mathfrak{X}, f \in H^{p+1}(\mathfrak{Y})$ существует единственное оптимальное управление $v \in H_{\partial}^{p+1}(\mathfrak{U})$ задачи (1)-(3), (5).

До ка з а тель ст в о. Зафиксируем $x_{0} \in \mathfrak{X}, f \in H^{1}(\mathfrak{Y})$, и рассмотрим (4) как отображение $G: u \rightarrow x(u)$. В условиях предыдущей теоремы отображение $G: H^{p+1}(\mathfrak{U}) \rightarrow H^{1}(\mathfrak{X})$, определенное формулой $(4)$, непрерывно. Теперь перепишем функционал качества (3) в виде

$$
J(u)=\left\|C x(t ; u)-z_{d}\right\|_{H^{1}(\mathfrak{Z})}^{2}+[\eta, u],
$$

где $\eta^{(k)}(t)=N_{k} u^{(k)}, k=0,1, \ldots, p+1$. Откуда

$$
J(u)=\pi(u, u)-2 \lambda(u)+\left\|z_{d}-C x(t ; 0)\right\|_{H^{1}(\mathfrak{Z})}^{2},
$$


где

$$
\pi(u, u)=\|C(x(t ; u)-x(t ; 0))\|_{H^{1}(\mathfrak{Z})}^{2}+[\eta, u]
$$

— билинейная непрерывная коэрцитивная форма на $H^{1}(\mathfrak{U})$, а

$$
\lambda(u)=\left\langle z_{d}-C x(t ; 0), C(x(t ; u)-x(t ; 0))\right\rangle_{H^{1}(\mathfrak{Z})}
$$

- линейная непрерывная на $H^{1}(\mathfrak{U})$ форма. А значит, утверждение теоремы следует из [7, гл. 1].

СледствиЕ. Пусть оператор $M$ сильно $(L, p)$-радиален, $p \in \mathbb{N}_{0}$, функиия $a \in C^{p+1}\left(\overline{\mathbb{R}}_{+} ; \mathbb{R}_{+}\right)$. Тогда при любых $x_{0} \in \mathfrak{X}, f \in H^{p+1}(\mathfrak{Y})$ существует единственное жёсткое управление $v \in H_{\partial}^{p+1}(\mathfrak{U})$ задачи (1)-(3), (5).

\section{СПИСОК ЛИТЕРАТУРЫ/ REFERENCES}

1. Г. А. Свиридюк, С. А. Загребина, "Задача Шоуолтера-Сидорова как феномен уравнений соболевского типа" // Известия Иркутского государственного университета. Сер. Математика, 2010. T. 3, №1. C. 104-125. [G. A. Sviridyuk, S. A. Zagrebina, "The Showalter-Sidorov problem as a phenomena of the Sobolev-type equations", Izvestiya Irkutskogo Gosudarstvennogo Universiteta. Ser. Matematika, 2010, vol. 3, no. 1, pp. 104-125 (In Russian)].

2. Г. В. Демиденко, С. В. Успенский, Уравнения и системы, не разрешенные относительно старшей производной. Новосибирск: Научная книга, 1998. xviii+437 c. [G. V. Demidenko, S. V. Uspenskiy, Uravneniya i sistemy, ne razreshennyye otnositel'no starshey proizvodnoy [Equations and systems that are not solved with respect to the highest derivative], Novosibirsk, Nauchnaya Kniga Publ., 1998, xviii+437 pp.]

3. G. A. Sviridyuk, V. E. Fedorov, Linear Sobolev type equations and degenerate semigroups of operators, Inverse and Ill-Posed Problems Series, Utrecht, Boston, VSP, 2003, viii+216 pp.

4. A. B. Al'shin, M. O. Korpusov, A. G. Sveshnikov, Blow-up in nonlinear Sobolev type equations, De Gruyter Series in Nonlinear Analysis and Applications, vol. 15, Berlin, Walter de Gruyter \& Co., 2011, xii+648 pp.

5. М. А. Сагадеева, А. Н. Шулепов, “Аппроксимации вырожденных $C_{0}$-полугрупп" // Вестник ЮУрГУ. Сер. Математическое моделирование и программирование, 2013. T. 6, № 2. C. 133-137. [M. A. Sagadeyeva, A. N. Shulepov, "The Approximations for Degenerate $C_{0}$-semigroup", Bulletin of the South Ural State University. Ser. Mathematical Modelling, Programming \& Computer Software, 2013, vol. 6, no. 2, pp. 133-137 (In Russian)].

6. М. А. Сагадеева, А. Д. Бадоян, "Оптимальное управление решениями нестационарных уравнений соболевского типа специального вида в относительно секториальном случае" // Вестник Магнитогорского государственного университета. Математика, 2013. № 15. C. 68-80. [M. A. Sagadeyeva, A. D. Badoyan, "The Optimal Control over Solutions of Special Form of Nonstacionary Sobolev Type Equations in Relatively Spectral Case", Vestnik Magnitogorskogo Gosudarstvennogo Universiteta. Matematika, 2013, no. 15, pp. 6880 (In Russian)].

7. J. L. Lions, Control of distributed singular systems, Paris, Gauther-Villars, 1985, 552 pp.; New York, John Wiley \& Sons Inc., 1987, 576 pp.; Ж. Л. Лионс, Управление сингулярными распределенными системами. М.: Наука, 1987. 368 с.

Поступила в редакцию 23/XII/2013;

в окончательном варианте - 12/I/2014;

принята в печать $-21 / \mathrm{II} / 2014$. 
MSC: 49J27; 47D06

\title{
PROBLEMS OF OPTIMAL AND HARD CONTROL OVER SOLUTIONS OF SPECIAL TYPE OF NONSTATIONARY SOBOLEV TYPE EQUATIONS
}

\author{
M. A. Sagadeeva, A. N. Shulepov \\ South Ural State University (National Research University), \\ 76, Lenin av., Chelyabinsk, 454080, Russian Federation.
}

\begin{abstract}
Sobolev type equations now constitute a vast area of nonclassical equations of mathematical physics. Those called nonclassical equations of mathematical physics, whose representation in the form of equations or systems of equations partial does not fit within one of the classical types (elliptic, parabolic or hyperbolic). In this paper we prove the existence of a unique optimal and hard control over solutions of Showalter-Sidorov problem for nonstationary operator-differential equations unresolved with respect to the time derivative. In this case, one of the operators in the equation is multiplied by a scalar function of the time-variable, besades stationary equation has a strong continuous degenerate resolving semigroup of operators. Apart from the introduction and bibliography article comprises two parts. The first part provides the necessary information regarding the theory of p-radial operators, the second contains the proof of main results of this article.
\end{abstract}

Keywords: optimal control, hard control, nonstationary Sobolev type equations, relatively radial case.

Received 23/XII/2013;

received in revised form $12 / \mathrm{I} / 2014$;

accepted $21 / \mathrm{II} / 2014$.

ISSN: 2310-7081 (online), 1991-8615 (print); doi: http://dx.doi.org/10.14498/vsgtu1286 (C) 2014 Samara State Technical University.

Citation: M. A. Sagadeeva, A. N. Shulepov, "Problems of Optimal and Hard Control over Solutions of Special Type of Nonstationary Sobolev Type Equations", Vestn. Samar. Gos. Tekhn. Univ., Ser. Fiz.-Mat. Nauki [J. Samara State Tech. Univ., Ser. Phys. \& Math. Sci.], 2014, no. 2 (35), pp. 33-38. doi: 10.14498/vsgtu1286. (In Russian)

Authors Details: Minzilia A. Sagadeeva (Cand. Phys. \& Math. Sci.), Associate Professor, Dept. of Information-Measuring Technique. Andrey N. Shulepov, Graduate Student, Dept. of Mathematical Physics Equations.

E-mail addresses: sagadeeva_ma@mail.ru (M.A. Sagadeeva, Corresponding author), andrewn92@mail.ru (A.N. Shulepov) 